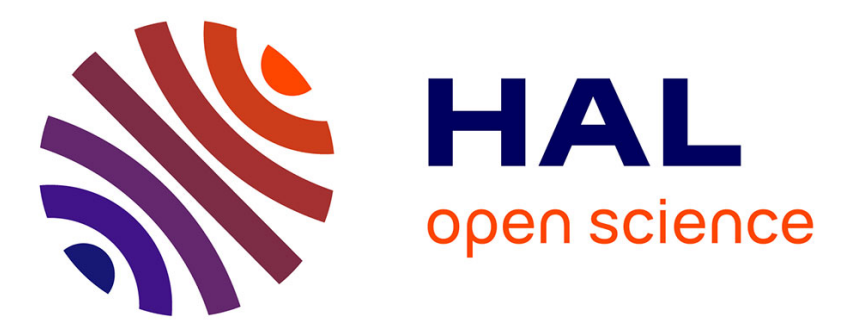

\title{
Anisotropy factor reconstruction as a new endogenous contrast for cancer diagnosis with optical tomography
}

Ahmad Addoum, Sylvain Contassot-Vivier, Fatmir Asllanaj

\section{To cite this version:}

Ahmad Addoum, Sylvain Contassot-Vivier, Fatmir Asllanaj. Anisotropy factor reconstruction as a new endogenous contrast for cancer diagnosis with optical tomography. Optical Tomography and Spectroscopy of Tissue XIII, Feb 2019, San Francisco, France. pp.68, 10.1117/12.2507418 . hal03522823

\section{HAL Id: hal-03522823 \\ https://hal.science/hal-03522823}

Submitted on 12 Jan 2022

HAL is a multi-disciplinary open access archive for the deposit and dissemination of scientific research documents, whether they are published or not. The documents may come from teaching and research institutions in France or abroad, or from public or private research centers.
L'archive ouverte pluridisciplinaire HAL, est destinée au dépôt et à la diffusion de documents scientifiques de niveau recherche, publiés ou non, émanant des établissements d'enseignement et de recherche français ou étrangers, des laboratoires publics ou privés. 


\title{
Anisotropy factor reconstruction as a new endogenous contrast for cancer diagnosis with optical tomography
}

\author{
Ahmad Addoum $^{\mathrm{a}}$, Sylvain Contassot-Vivier ${ }^{\mathrm{b}}$, and Fatmir Asllanaj ${ }^{\mathrm{a}}$ \\ ${ }^{a}$ University of Lorraine, ENSEM, LEMTA, F-54518, Nancy, France \\ ${ }^{\mathrm{b}}$ University of Lorraine, LORIA, F-54506, Nancy, France
}

\begin{abstract}
In optical tomography, the reconstructions have only been limited to the absorption $\mu_{a}$ and scattering $\mu_{s}$ coefficients of biological tissues due to theoretical and computational limitations. In this study, The authors propose an efficient method to reconstruct, in 3D geometries, the anisotropy factor $g$ of the Henyey-Greenstein phase function as a new optical contrast for cancer diagnosis. The light propagation in biological tissues is accurately modeled by the Radiative Transfer Equation (RTE) in the frequency domain. The adjoint method is used to efficiently compute the gradient of the objective function. A parallel implementation is carried out to reduce the computational times. The results show the robustness of the algorithm to reconstruct the $g$-factor for different contrast levels and for different initial guesses. The crosstalk problem between $\mu_{s}$ and $g$ has been achieved with a reasonable quality which makes the new algorithm a candidate of choice to image this factor as new intrinsic contrast for optical imaging.
\end{abstract}

Keywords: Optical tomography, scattering phase function, cancer diagnosis, radiative transfer, anisotropy factor, adjoint method.

\section{INTRODUCTION}

Diffuse Optical Tomography (DOT) is a non-invasive imaging modality that aims to reconstruct the optical properties of biological tissues namely, the absorption $\mu_{a}$ and the scattering $\mu_{s}$ coefficients. It is well known that these tissues are highly forward scattering media where the angular distribution can be described by the HenyeyGreenstein (H-G) phase function. The mean value of this function is represented by the anisotropy factor $g$ which characterize the anisotropic scattering. Previous studies ${ }^{1-3}$ have shown that this factor can be modified when the tissue is affected by a tumor. Consequently, the $g$-factor can provide additional morphological and pathological information for optical imaging. Nevertheless, and to the best of our knowledge, the reconstruction of this factor has not been carried out up to now due to theoretical and computational requirements. Indeed, it requires an accurate forward model of light propagation which takes into account the anisotropic scattering behavior. This can be insured with the Radiative Transfer Equation (RTE) which has been addressed as a rigorous model for light transport in biological tissues. It should be noted that the diffusion equation is useless in this case since it's devoted to describing an isotropic fluence field. In DOT, another challenge is the reconstruction technique used to iteratively update the spatial distribution of optical properties. The gradient-based scheme ${ }^{4,5}$ is well suitable for DOT but the computation of the objective function gradient remains a crucial step. In order to efficiently evaluate the gradient, the adjoint method has been developed to compute it regardless the number of unknowns throughout a Lagrangian formalism. It must be kept in mind that the RTE solution requires complex computational methods which are expensive in terms of runtime and memory resources. Thus, reconstructing the anisotropy factor $g$, in 3D geometries, is daunting and prohibitively expensive with the RTE, especially in this case where the phase function becomes spatial and angular dependent and must be evaluated at each iteration in the inversion procedure. To overcome this difficulty, different levels of parallelization were carried out within our reconstruction algorithm. The remainder of this paper is organized as follows. In Sec.2, the RTE model is presented and the predictions on semi-transparent boundaries are given. In Sec. 3, the parallel reconstruction algorithm based on the Lagrangian formalism is described. Reconstructions results based on synthetic data are presented and discussed in Sec. 4. Concluding remarks are finally offered in Sec. 5.

Further author information: (Send correspondence to Ahmad Addoum)

E-mail: Ahmad.addoum@univ-lorraine.fr,

Telephone: +33(0)78171 7707 


\section{FORWARD MODEL}

The RTE in the frequency-domain is used to accurately described the light propagation within biological tissues. The external Laser beam $\Gamma\left(\boldsymbol{r}_{\boldsymbol{s}}, \omega\right)$ illuminating the surface at the position $\boldsymbol{r}_{\boldsymbol{s}}$ with the angular modulation frequency $\omega$ is seperated into two components $\psi=\psi_{c}+\psi_{s}$, respectively the collimated $\psi_{c}$ and scattered radiance $\psi_{s}$. This allows taking into account the collimated radiation in our forward model. The $\psi_{c}$ component is governed by the RTE state equation denoted $\mathcal{R}_{c}$ in the collimated direction $\boldsymbol{\Omega}_{\boldsymbol{c}}$ and is analytically solved in accordance with the Bouguer-Beer-Lambert exponential law :

$$
\mathcal{R}_{c}=\left[\boldsymbol{\Omega}_{\boldsymbol{c}} \cdot \nabla+\left(\frac{i n \omega}{c}+\mu_{t}(\boldsymbol{r})\right)\right] \psi_{c}(\boldsymbol{r}, \omega)=0
$$

where $c$ is light velocity in vacuum while $n$ is the refractive index of tissue. The total extinction coefficient $\mu_{t}(\boldsymbol{r})$, at position $\boldsymbol{r}$, represents the sum of the absorption $\mu_{a}(\boldsymbol{r})$ and the scattering $\mu_{s}(\boldsymbol{r})$ coefficients. The tissue surfaces are modeled as semi-transparent boundaries due to the refractive index mismatch between air and tissue. The boundary condition for the collimated radiance is thus given by :

$$
\psi_{c}\left(\boldsymbol{r}_{\boldsymbol{s}}, \omega\right)=\left[1-R\left(\Theta^{\prime}\right)\right] \Gamma\left(\boldsymbol{r}_{\boldsymbol{s}}, \omega\right) \text { for } \boldsymbol{\Omega}_{\boldsymbol{c}} \cdot \boldsymbol{n}_{\text {out }}<0,
$$

where $\boldsymbol{n}_{\text {out }}$ is the local outward unit vector normal to the tissue boundary. The reflectivity coefficient $R$ depends on the incidence angle $\Theta^{\prime}$, with $\cos \Theta^{\prime}=\boldsymbol{\Omega}^{\prime} \cdot \boldsymbol{n}_{\text {out }}$, and where the incident direction $\boldsymbol{\Omega}^{\prime}$ satisfied $\boldsymbol{\Omega}^{\prime} \cdot \boldsymbol{n}_{\text {out }}>0$. Once the $\mathcal{R}_{c}$ equation is solved, the solution $\psi_{c}$ is then used through a source term $S_{c}$ such as :

$$
S_{c}(\boldsymbol{r}, \boldsymbol{\Omega}, \omega)=\mu_{s}(\boldsymbol{r}) p\left(\boldsymbol{r}, \boldsymbol{\Omega}_{\boldsymbol{c}} \cdot \boldsymbol{\Omega}\right) \psi_{c}(\boldsymbol{r}, \omega)
$$

where $p\left(\boldsymbol{r}, \boldsymbol{\Omega}^{\prime} \cdot \boldsymbol{\Omega}\right)$ is the Henyey-Greenstein (H-G) phase function which represents the probability that photon traveling in incident direction $\boldsymbol{\Omega}^{\prime}$ deviates into scattering direction $\boldsymbol{\Omega}$. Its mathematical expression in 3D participating media ${ }^{6}$ is given by :

$$
p\left(\boldsymbol{r}, \boldsymbol{\Omega}^{\prime} \cdot \mathbf{\Omega}\right)=\frac{1}{4 \pi} \frac{1-g^{2}(\boldsymbol{r})}{\left(1+g^{2}(\boldsymbol{r})-2 g(\boldsymbol{r}) \boldsymbol{\Omega}^{\prime} \cdot \boldsymbol{\Omega}\right)^{3 / 2}}
$$

where $g$ is the anisotropy factor characterizing the angular distribution of tissue scattering. In this study, $g$ is space-dependent. Hence, further the dependence of the phase function $p$ on the incident and scattered directions through an inner product, $p$ is also a function of the spatial position $\boldsymbol{r}$ (see Eq. 4). The coupling term $S_{c}$ (Eq. 3 ) is included within the RTE state equation $\mathcal{R}_{s}$ which governs the scattered radiance $\psi_{s}$ in the direction $\boldsymbol{\Omega}$ :

$$
\begin{array}{r}
\mathcal{R}_{s}=\left[\boldsymbol{\Omega} \cdot \nabla+\left(\frac{i n \omega}{c}+\mu_{t}(\boldsymbol{r})\right)\right] \psi_{s}(\boldsymbol{r}, \boldsymbol{\Omega}, \omega) \\
-\mu_{s}(\boldsymbol{r}) \int_{\Omega^{\prime}=4 \pi} p\left(\boldsymbol{r}, \boldsymbol{\Omega}^{\prime} \cdot \boldsymbol{\Omega}\right) \psi_{s}\left(\boldsymbol{r}, \boldsymbol{\Omega}^{\prime}, \omega\right) d \Omega^{\prime}-S_{c}(\boldsymbol{r}, \boldsymbol{\Omega}, \omega)=0
\end{array}
$$

The reflectance signals correspond to the outgoing flux predicted at the detector position $\boldsymbol{r}_{\boldsymbol{d}}$ on the illuminated bounding surface such as :

$$
P\left(\boldsymbol{r}_{\boldsymbol{d}}, \omega\right)=\int_{\boldsymbol{\Omega}^{\prime} \cdot \boldsymbol{n}_{\text {out }}>0}\left[1-R\left(\Theta^{\prime}\right)\right] \psi_{s}\left(\boldsymbol{r}_{\boldsymbol{d}}, \boldsymbol{\Omega}^{\prime}, \omega\right)\left(\boldsymbol{\Omega}^{\prime} \cdot \boldsymbol{n}_{\text {out }}\right) d \Omega^{\prime}
$$

In our previous publication, ${ }^{7}$ we developed a modified finite volume method (MFVM) to solve the 2D RTE. The angular space was uniformly subdivided into several discrete directions. The "modified" word is due to the fact the photon transport is solved even inside each control volume within an exponential scheme. The MFVM is thus qualified as a highly accurate method compared to other deterministic numerical methods available in the literature. Excellent agreement was found with relative differences less than 1\% when the MFVM solutions were compared against to those of Monte-Carlo and analytical solutions from simple cases. ${ }^{7}$ The MFVM was extended to 3D geometries. The methodology of the employed method is not repeated here, we refer the reader to [7] for comprehensive details. 


\section{INVERSE PROBLEM}

\subsection{Lagrangian formalism and adjoint model}

The inverse problem can be considered as a large-scale optimization problem which consists to minimize the least-square discrepancy between the measurements $M\left(\boldsymbol{r}_{d}, \omega\right)$ and the forward model predictions $P\left(\boldsymbol{r}_{d}, \omega\right)$ at the detector position $\boldsymbol{r}_{d}$ and angular frequency $\omega$. These discrepancies are represented by a real-valued non-negative function, called the objective function defined by :

$$
\mathcal{J}(\beta, \omega)=\sum_{d=1}^{N_{d}}\left\|P\left(\boldsymbol{r}_{d}, \omega\right)-M\left(\boldsymbol{r}_{d}, \omega\right)\right\|_{2}^{2}
$$

where $\|\cdot\|_{2}$ denotes Euclidean norm. The vector $\beta$ contains the spatial distribution of optical properties to be reconstruct and $N_{d}$ is the total number of detectors on the tissue surface. The objective is to determine the vector $\hat{\beta}$ that minimizes this function and vanishes the gradient $\nabla \mathcal{J}(\hat{\beta}, \omega)=0$. In this work, instead of solving the unconstrained problem (Eq. 7), we adopted an adjoint method that solves the constrained optimization problem by following the Lagrangian formalism given as

$$
\mathcal{L}\left(\beta, \psi_{s}, \psi_{c}, \lambda_{s}, \lambda_{c}\right)=\mathcal{J}(\beta, \omega)+\left\langle\lambda_{s} \mid \mathcal{R}_{s}\left(\beta, \psi_{s}, \psi_{c}\right)\right\rangle_{s}+\left\langle\lambda_{c} \mid \mathcal{R}_{c}\left(\beta, \psi_{c}\right)\right\rangle_{c}
$$

where $\lambda_{s}$ and $\lambda_{c}$ are respectively the Lagrange multipliers to $\psi_{s}$ and $\psi_{c} \cdot\langle\cdot \mid \cdot\rangle_{s}$ and $\langle\cdot \mid \cdot\rangle_{c}$ denote the inner products associated to the solution space of $\psi_{s}$ and $\psi_{c}$, respectively. ${ }^{8}$ The objective function (Eq. 7) is minimized under some equality constraints based on the RTE state equations. The optimization problem corresponds to find the stationary point (vector solution $\hat{\beta}$ ) of the Lagrangian function (Eq. 8) that vanishes its gradient $\nabla \mathcal{L}$ with respect to all its variables $\nabla_{\lambda_{s, c}} \mathcal{L}=\nabla_{\psi_{s, c}} \mathcal{L}=\nabla_{\beta} \mathcal{L}=0$. Using the adjoint operator and the inner product properties, the differenciation of $\mathcal{L}$ with respect to the state variables $\psi_{s}$ and $\psi_{c}\left(\nabla_{\psi_{s, c}} \mathcal{L}\right)$ leads to the RTE adjoint equations for the scattered and collimated components, respectively :

$$
\begin{gathered}
{\left[-\boldsymbol{\Omega} \cdot \nabla+\left(\frac{-i \omega}{c / n}+\mu_{t}(\boldsymbol{r})\right)\right] \lambda_{s}(\boldsymbol{r}, \boldsymbol{\Omega}, \omega)=\mu_{s}(\boldsymbol{r}) \int_{4 \pi} p\left(\boldsymbol{r}, \boldsymbol{\Omega}^{\prime} \cdot \boldsymbol{\Omega}\right) \lambda_{s}\left(\boldsymbol{r}, \boldsymbol{\Omega}^{\prime}, \omega\right) d \mathbf{\Omega}^{\prime}-\frac{\partial \mathcal{J}(\boldsymbol{\beta}, \omega)}{\partial \psi_{s}}} \\
{\left[-\boldsymbol{\Omega}_{c} \cdot \nabla+\left(\frac{-i \omega}{c / n}+\mu_{t}(\boldsymbol{r})\right)\right] \lambda_{c}(\boldsymbol{r}, \omega)=\mu_{s}(\boldsymbol{r}) \int_{4 \pi} p\left(\boldsymbol{r}, \boldsymbol{\Omega}_{\boldsymbol{c}} \cdot \boldsymbol{\Omega}\right) \lambda_{s}(\boldsymbol{r}, \boldsymbol{\Omega}, \omega) d \boldsymbol{\Omega}}
\end{gathered}
$$

Note that $\nabla_{\psi_{c}} \mathcal{J}(\beta, \omega)=0$ and $\nabla_{\beta} \mathcal{J}(\beta, \omega)=0$, because the objective function is independent on the collimated light and it does not vary explicitly with respect to $\beta$. The Lagrange multipliers $\lambda_{s}$ and $\lambda_{c}$ are solutions to (Eq. 9) and (Eq. 10), respectively. The above system was derived in details in our previous study ${ }^{8}$ based on $2 \mathrm{D}$ geometries. Note that, in $3 \mathrm{D}$ geometries, the integral term over $2 \pi$ is replaced by the integral term over $4 \pi$. One major advantage of this approach is its simple implementation by applying the same numerical solver as used for the forward model. Furthermore, the adjoint method provides the gradient of the objective function in a fast and efficient way (independently of the number of unknown parameters). Once the adjoint equations are solved and the Lagrange multipliers are obtained, these last are then used to compute the gradient $\left(\nabla_{\beta} \mathcal{J}\right)$ with a computational time equivalent to a one forward simulation.

\subsection{Gradient expressions}

Differentiating now the functional $\mathcal{L}$ with respect to $\mu_{a}, \mu_{s}$ and $g$, we obtain the gradient expressions with respect to the optical properties such as :

$$
\begin{array}{r}
\nabla_{\mu_{a}} \mathcal{J}(\boldsymbol{r}, \omega)=\mathfrak{R e} \int_{\boldsymbol{\Omega}=4 \pi} \overline{\lambda_{s}(\boldsymbol{r}, \boldsymbol{\Omega}, \omega)} \psi_{s}(\boldsymbol{r}, \boldsymbol{\Omega}, \omega) d \boldsymbol{\Omega}+\overline{\lambda_{c}(\boldsymbol{r}, \omega)} \psi_{c}(\boldsymbol{r}, \omega) \\
\nabla_{\mu_{s}} \mathcal{J}(\boldsymbol{r}, \omega)=\nabla_{\mu_{a}} \mathcal{J}(\boldsymbol{r}, \omega)-\mathfrak{R e} \int_{\boldsymbol{\Omega}=4 \pi} \overline{\lambda_{s}(\boldsymbol{r}, \boldsymbol{\Omega}, \omega)}\left(\int_{\boldsymbol{\Omega}^{\prime}=4 \pi} \psi_{s}\left(\boldsymbol{r}, \boldsymbol{\Omega}^{\prime}, \omega\right) p\left(\boldsymbol{r}, \boldsymbol{\Omega}^{\prime} \cdot \boldsymbol{\Omega}\right) d \Omega^{\prime}+\right. \\
\left.\psi_{c}(\boldsymbol{r}, \omega) p\left(\boldsymbol{r}, \boldsymbol{\Omega}_{c} \cdot \boldsymbol{\Omega}\right)\right) d \boldsymbol{\Omega}
\end{array}
$$




$$
\begin{array}{r}
\nabla_{g} \mathcal{J}(\boldsymbol{r}, \omega)=-\mathfrak{R e} \int_{\boldsymbol{\Omega}=4 \pi} \frac{\overline{\lambda_{s}(\boldsymbol{r}, \boldsymbol{\Omega}, \omega)} \mu_{s}(\boldsymbol{r})\left(\psi_{c}(\boldsymbol{r}, \omega) \frac{\partial p\left(\boldsymbol{r}, \boldsymbol{\Omega}_{c} \cdot \boldsymbol{\Omega}\right)}{\partial g}+\right.}{\left.\int_{\boldsymbol{\Omega}^{\prime}=4 \pi} \psi_{s}\left(\boldsymbol{r}, \boldsymbol{\Omega}^{\prime}, \omega\right) \frac{\partial p\left(\boldsymbol{r}, \boldsymbol{\Omega}^{\prime} \cdot \boldsymbol{\Omega}\right)}{\partial g} d \Omega^{\prime}\right) d \boldsymbol{\Omega}}
\end{array}
$$

The derivative of the H-G phase function with respect to the $g$-factor is evaluated analytically. At each inversion iteration, the forward and adjoint models are solved and the gradient is accurately obtained accordingly to the above expressions. This last is then used within a reconstruction scheme based on the Limited-memory BFGS method to iteratively update the spatial distribution of the optical properties. The reconstruction of the anisotropy factor $g$ in 3D media is expected to be extremely expensive in terms of time and memory since the H-G phase function $p$ depends on $\boldsymbol{r}=(x, y, z), \boldsymbol{\Omega}^{\prime}$ and $\boldsymbol{\Omega}$. In order to make the $g$ reconstruction possible, parallel implementation in the present algorithm was unavoidable.

\subsection{Parallel computing}

It is clear that exploiting the RTE model, within an iterative procedure, to reconstruct the optical properties for 3D geometries can take long computational times. Then, we implemented a multi-threading parallel algorithm. At this level, multiple threads compute the RTE along different directions. It should be pointed out that the $\mathrm{H}-\mathrm{G}$ phase function is calculated only once when the medium is assumed to be homogeneous in $g$-factor. On the other hand, when this factor has to be reconstructed ( $g$-factor varies spatially inside the medium), the $\mathrm{H}$ G phase function and also its derivative (required for the gradient $\nabla_{g} \mathcal{J}$ ) must be evaluated at every spatial node and overall incident and scattered directions of light propagation. Further the extensive computational times, the resulting values necessitate huge memory space which may exceed the capability of a single machine. Consequently, reconstructing the spatial distribution of this factor in 3D media is strongly restricted when using a single machine. In that regard, our algorithm was extended to support multiple machines, via explicit Messages Passing Interface (MPI). In this context, some of the data were distributed over the machines of the computing system and data exchanges were performed between them for the computations that require all the data. It is worth noticing that the use of parallelism was limited to some parts of our algorithm that consist to solve the integral term over the $\boldsymbol{\Omega}$ directions in the RTE model. Indeed, those parts are the most time consuming and their parallelization provides most of the theoretical speed gain that can be expected according to the sequential version. The parallel application, using OpenMP and MPI libraries, is designed to efficiently use classical clusters of multi-core machines. Additional studies are currently conducted to see how GPU accelerators may be efficiently exploited in our reconstruction algorithm.

\section{RESULTS AND DISCUSSION}

\subsection{Model description}

It is known that the light propagation becomes isotropic $(g=0)$ when the photons have traveled several transport mean-free path in the medium $\left(\approx 8 \times l_{t r}\right) .{ }^{10}$ In order to take into consideration the anisotropic scattering behavior, the reconstructed geometry of $g$ should not exceed approximately $8 \times l_{t r}$. In that regard, the three-dimensional numerical phantom used for reconstruction is a cube of length $4 \mathrm{~mm}$. It contains a spherical heterogeneity in the $g$-factor with $1 \mathrm{~mm}$ of a diameter centered at $X=1 \mathrm{~mm}$ in depth, $Y=Z=1 \mathrm{~mm}$. This last is embedded in a homogeneous background medium with $\mu_{a}=0.01 \mathrm{~mm}^{-1}, \mu_{s}=2 \mathrm{~mm}^{-1}$ and $g=0.8$. The source is modulated at $f=600 \mathrm{MHz}$ and positioned at $(0,0.5,0.5)$ on the western surface of the phantom. Only The backscattered light (reflectance) on the illuminated boundary is used for reconstruction.

The computational domain was discretized with 68,921 nodes and 384,000 tetrahedral elements for reconstruction. 320 detectors readings were generated on the western surface using a finer mesh of 746,691 nodes in order to avoid the inverse crime. The angular space $(4 \pi \mathrm{Sr})$ was uniformly discretized into 64 control solid angles and each angle was also subdivided into 8 azimuthal and polar directions for the phase function normalization. ${ }^{7}$

The generated data are contaminated with $1 \%$ of Gaussian random noise and given as an input in the reconstruction algorithm. The inversion procedure is started with an initial guess corresponding to the homogeneous background. It terminated after the normalized difference of the objective function between two subsequent 
iterations was smaller than a prescribed value, here equal to $10^{-5}$. The quality reconstruction was determined thanks to the relative error $\varepsilon$ between exact $\left(\beta^{*}\right)$ and estimated $(\hat{\beta})$ values such as :

$$
\varepsilon=100 \% \cdot \frac{\left\|\hat{\beta}-\beta^{*}\right\|_{2}}{\left\|\beta^{*}\right\|_{2}}
$$

\subsection{Different contrast levels}

The robustness of the algorithm is examined with two different contrast levels through two test cases. The first one considers a relatively high contrast inclusion with $+18 \%\left(g^{*}=0.95\right)$ while the second case corresponds to a low contrast level with $-6 \%\left(g^{*}=0.75\right)$ of discard with respect to the homogeneous background value $(g=$ 0.8 ). For the first test case, the minimization has been stopped after 10 iterations. The obtained $3 \mathrm{D}$ slices at the $x=1 \mathrm{~mm}, y=1 \mathrm{~mm}$ and $z=1 \mathrm{~mm}$ planes are plotted in the Figs. 1a-1c, respectively. The 1D reconstructed profiles passing through the center of the inclusion are depicted in Fig. 1d.

(a)

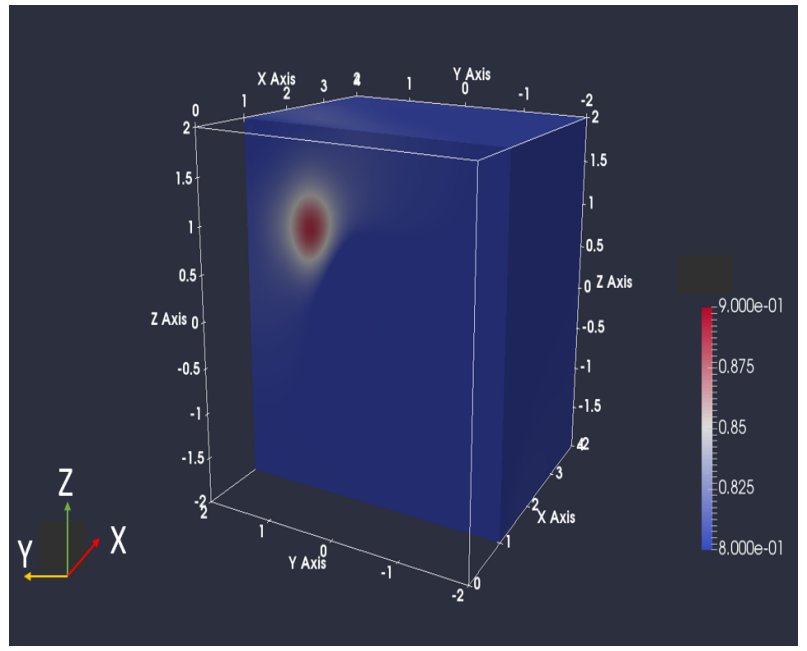

(c)

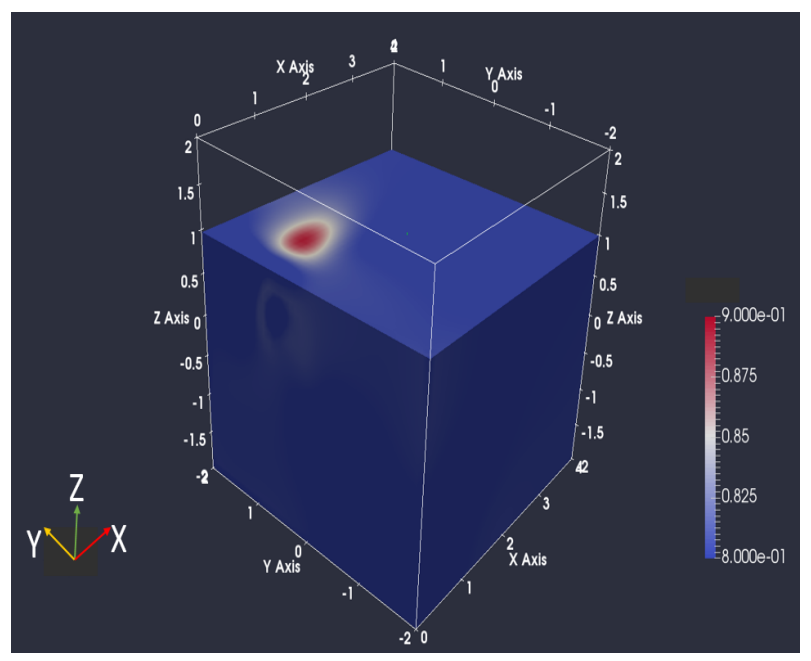

(b)

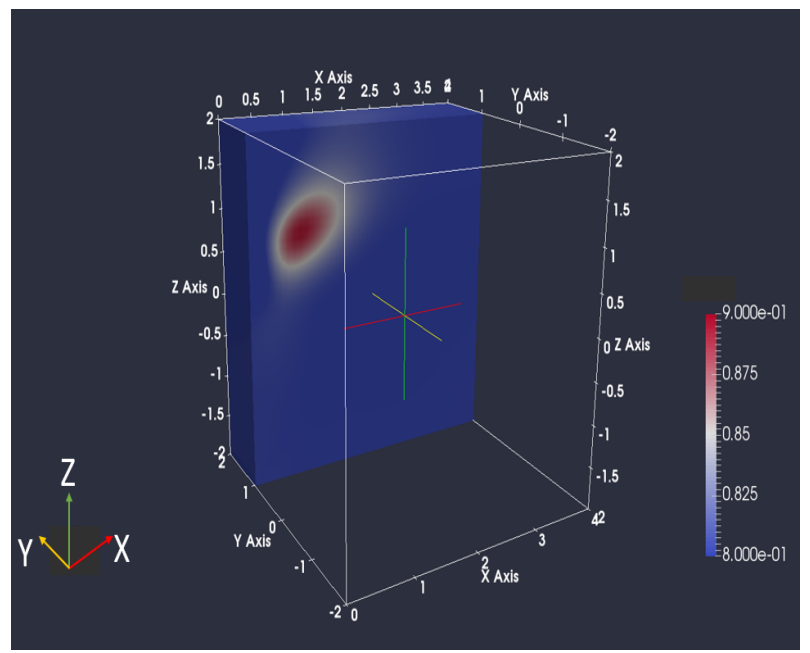

(d)

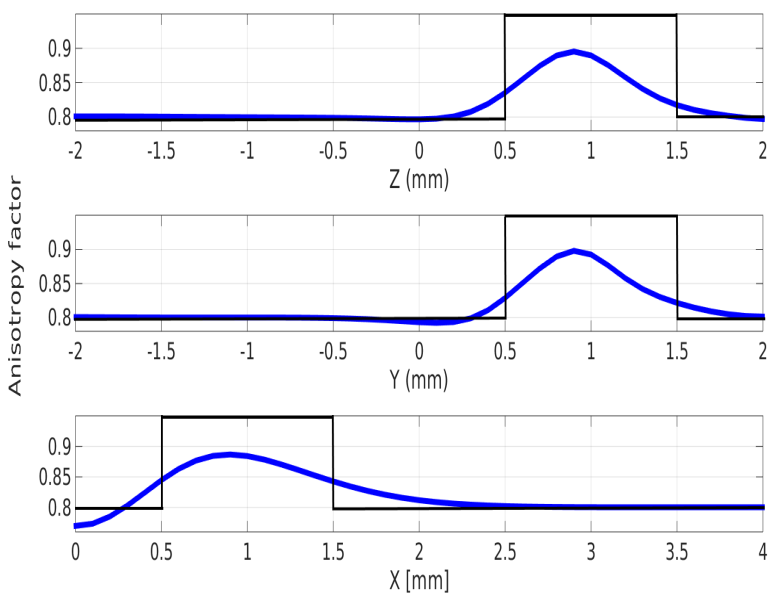

Figure 1: Reconstructed $g$-factor image obtained after convergence. (a), (b) and (c) display the 3D slices selected at $x=1 \mathrm{~mm}, y=1 \mathrm{~mm}$ and $z=1 \mathrm{~mm}$, respectively. (d) represents the $1 \mathrm{D}$ reconstructed profiles passing by the inclusion center along the $x-y$ - and $z$ - axis. 
It is readily seen that the inclusion has been located at approximately the right position $\left(x_{i n c}=0.9 \mathrm{~mm}, y_{\text {inc }}\right.$ $=0.9 \mathrm{~mm}, z_{i n c}=0.9 \mathrm{~mm}$ ) and the circular shape was clearly reconstructed. However, the maximum retrieved value inside the inclusion is somehow under-estimated compared to its original value with an error $\varepsilon=14.4 \%$ (Fig. 1d). As expected, the computation cost is expensive as each iteration in the inversion procedure has required $100 \mathrm{~min}$ to calculate the gradient. In order to assess the convergence quality of the algorithm, the 2D images in the $z=1 \mathrm{~mm}$ plane selected at iteration equal to 2, 4 and 6 are displayed in the Figs. 2a-c, respectively. Figure $2 \mathrm{~d}$ depicts the 1D reconstructed profiles along $y=1 \mathrm{~mm}$.

(a)

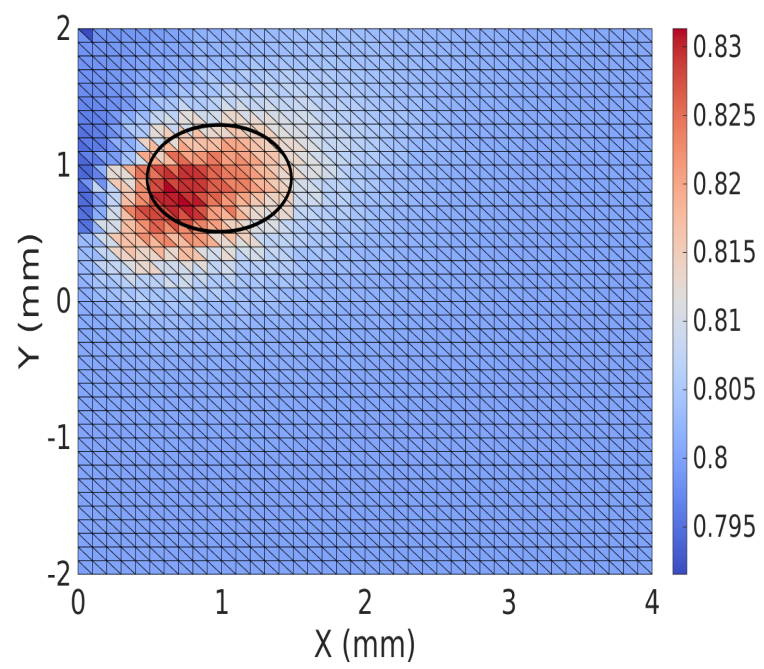

(c)

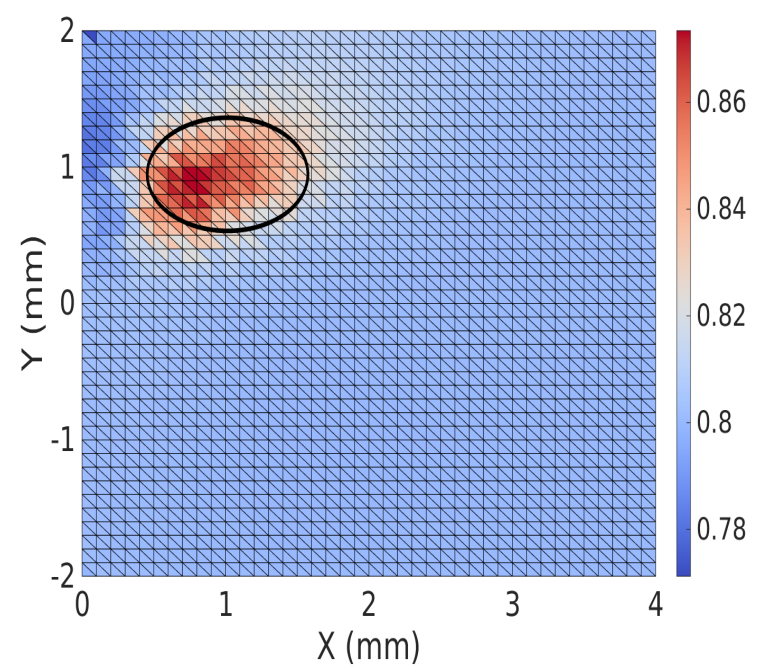

(b)

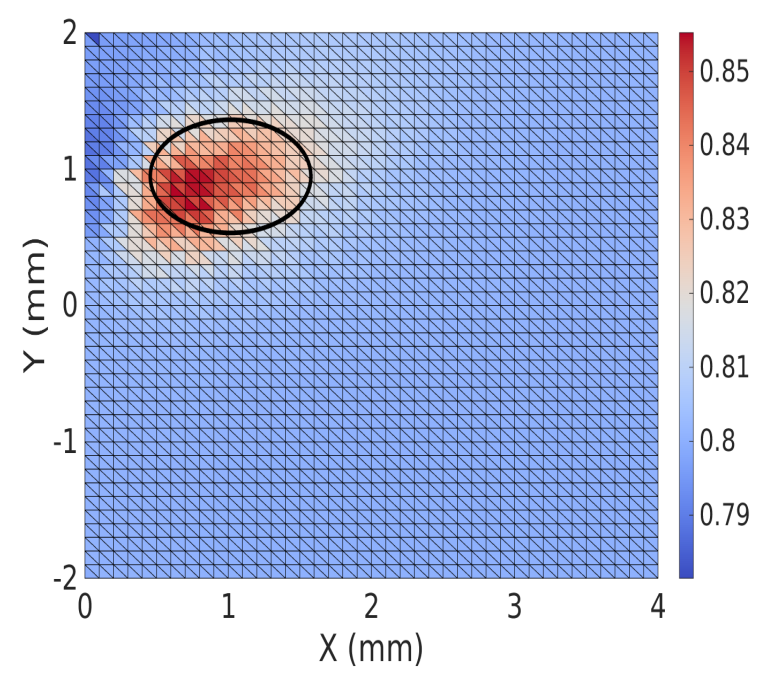

(d)

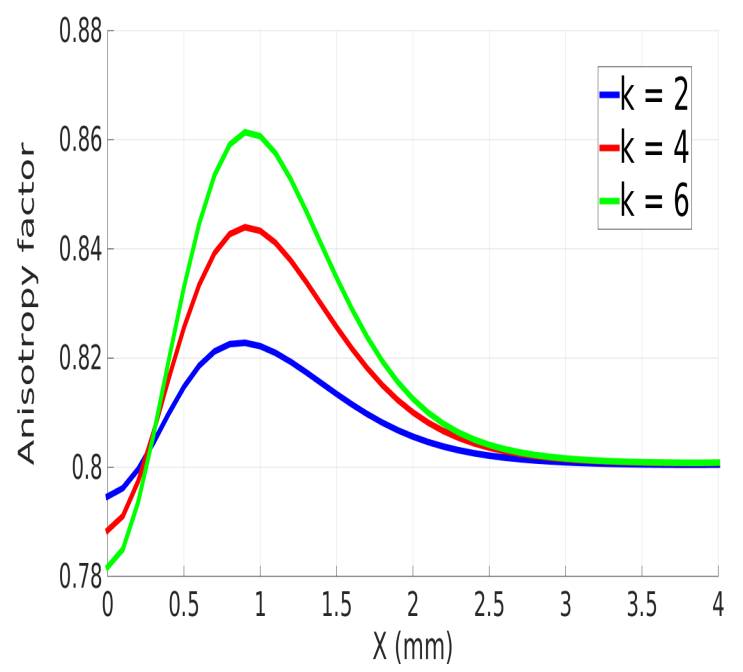

Figure 2: Reconstructed $g$-factor images obtained at different iteration $k$ of the inversion process. (a), (b) and (c) display the 2D $x y$ slices selected at $z=1 \mathrm{~mm}$ plane for $k=2, k=4$ and $k=6$, respectively. (d) represents the $1 \mathrm{D}$ reconstructed profiles passing by $y=1 \mathrm{~mm}$ and through the inclusion center for the three iterations.

The results show that the algorithm provides a good qualitative indication at early iterations where the heterogeneity has been already reconstructed and accurately localized from only the second iteration. The suspect area can then be defined very quickly (in a few minutes) and might be exploited as a prior structural knowledge. For the test case 2, the stopped criterion has been satisfied at the 17 th iteration and the corresponding 
results are posted in Figs. 3a-d. Figure 3d shows that the algorithm has spatially well fitted the retrieved values with the original solution along the $x$ - $y$ - and $z$ - axis.

(a)

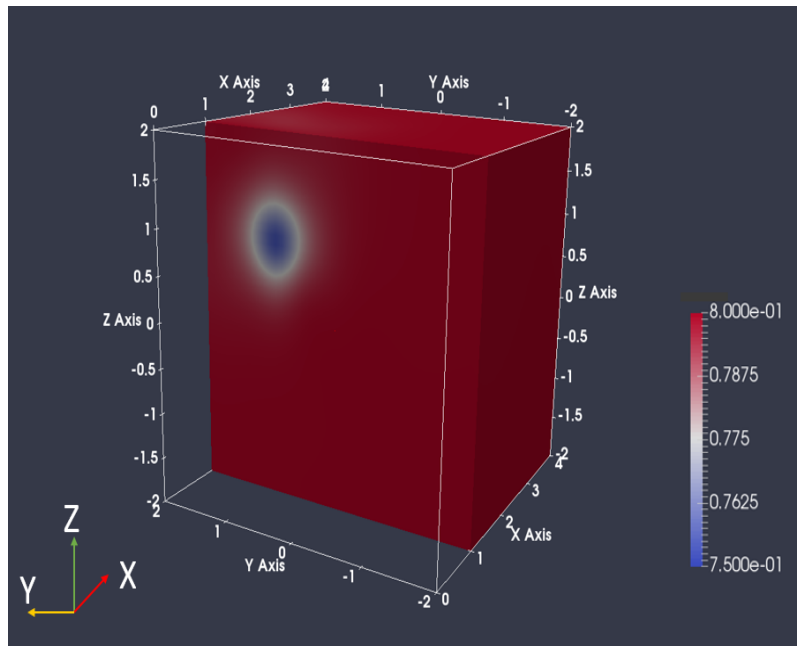

(c)

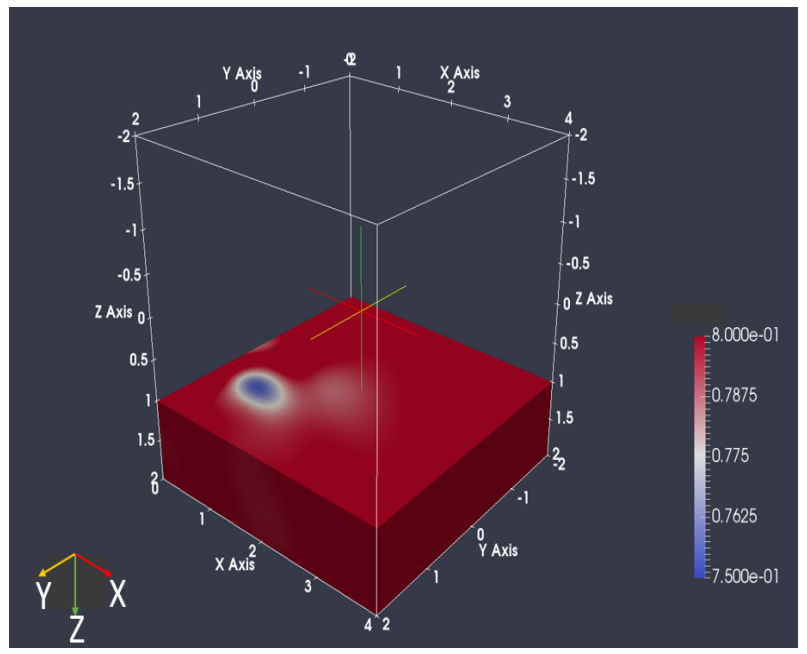

(b)

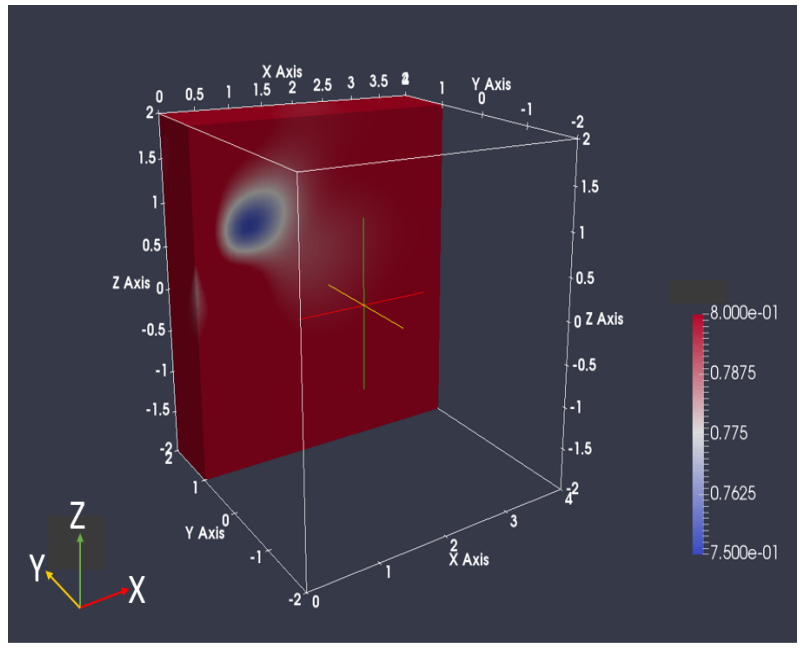

(d)

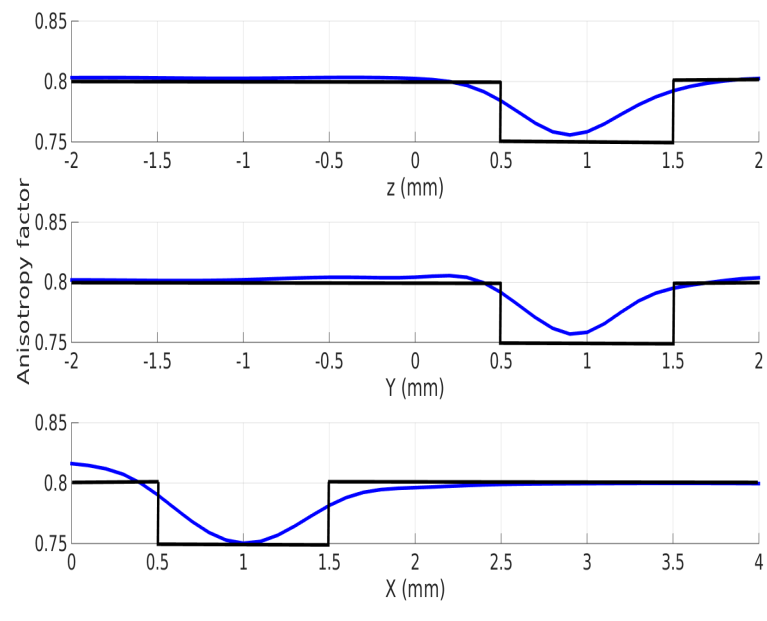

Figure 3: Reconstructed $g$-factor image obtained after convergence. (a), (b) and (c) display the 3D slices selected at $x=1 \mathrm{~mm}, y=1 \mathrm{~mm}$ and $z=1 \mathrm{~mm}$, respectively. (d) represents the $1 \mathrm{D}$ reconstructed profiles passing by the inclusion center along the $x-y$ - and $z$ - axis.

Moreover, the center of the inclusion has been located $\left(x_{i n c}=1 \mathrm{~mm}, y_{i n c}=0.9 \mathrm{~mm}, z_{i n c}=0.9 \mathrm{~mm}\right)$ with a good agreement with respect to the target position. Although the poor contrast level, the algorithm was able to reveal the small spatial heterogeneity in the medium. This is mainly due to the high sensitivity of the $g$-factor on the boundary data where a small variation of it can largely change the $\mathrm{H}-\mathrm{G}$ phase function. Compared to the previous test case, the quantitative accuracy has been improved and the relative estimation error of the inclusion has been decreased to $2.66 \%$. This is because the discrepancy between the initial value and the exact solution of the inverse problem is reduced when the contrast level is low which makes, therefore, the estimation more accurate. 


\subsection{Different initial guesses}

One of the gradient-based optimization schemes drawbacks is the choice of the initial estimate which should not be far away from the exact solution of the iterative procedure. Usually, this estimate is described by the homogeneous optical properties of the original background. To assess the robustness of the algorithm on different homogeneous initial guesses $g^{0}$, the previous test case $\left(g_{\text {inclusion }}^{*}=0.75\right)$ is reconstructed with two examples of initial guesses $g^{0}=0.82$ and $g^{0}=0.85$ which are different to the original background medium $\left(g_{\text {background }}^{*}=\right.$ 0.8). The retrieved profils along $x, y$ and $z$ axis are shown in figure 4 and compared the those of the previous test. The relative estimation errors of inclusion and background are listed in Table 1.

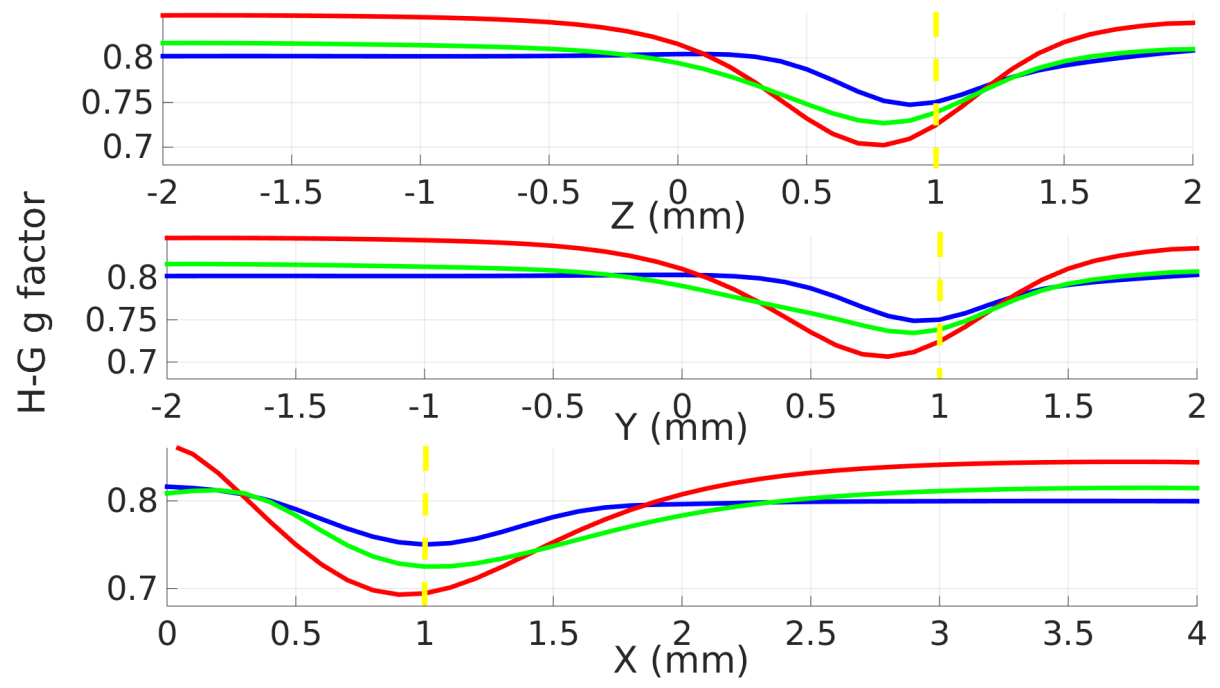

Figure 4: The reconstructed profiles passing through the center position of the inclusion along the $x, y$ and $z$ axis for the three different initial estimates. The red, green and blue profiles correspond to $g^{0}=0.85, g^{0}=0.82$ and $g^{0}=0.80$, respectively. The dashed yellow lines indicate the center position of the inclusion.

\begin{tabular}{c|ccc|cc}
\hline \multicolumn{3}{c}{ Inclusion } & \multicolumn{2}{c}{ Background } \\
\hline Initial Guess & $\varepsilon_{g}(\%)$ & $\varepsilon_{\text {position }}(\%)$ & $\hat{g}_{\text {minimum }}$ & $\varepsilon_{g}(\%)$ & $\hat{g}_{\text {average }}$ \\
\hline$g^{0}=0.8$ & $2.66 \%$ & $8.1 \%$ & 0.75 & $0.88 \%$ & 0.7981 \\
$g^{0}=0.82$ & $3.63 \%$ & $14.4 \%$ & 0.73 & $1.82 \%$ & 0.8131 \\
$g^{0}=0.85$ & $4.46 \%$ & $21.21 \%$ & 0.68 & $5.41 \%$ & 0.8420 \\
\hline
\end{tabular}

Table 1: The relative errors of background and inclusion for the three different initial guesses. $\hat{g}_{\text {minimum }}$ represents the minimum estimated value of $g$-factor and $\hat{g}_{\text {average }}$ denotes its estimated mean value.

Qualitatively, the algorithm has correctly found the spatial localization of the inclusion when the initial value was different from the original background value (see Fig. 4). The relative estimation errors of the $g$-factor increase from $2.66 \%$ to $4.46 \%$ for the inclusion and from $0.88 \%$ to $5.41 \%$ for the background when the homogeneous initial value $g^{0}$ has been chosen from 0.8 to 0.85 , respectively (see Tab. 1). Furthermore, the relative position errors of the inclusion increase and its retrieved values becomes more under-estimated (from $\hat{g}_{\text {minimim }}=0.75$ to $\left.\hat{g}_{\text {minimim }}=0.68\right)$ when the $g^{0}$ moves away from the original background value. Additionally, the retrieved mean values of the reconstructed background don't change sufficiently and remain near to the initial homogeneous value (see Tab. 1).

\subsection{Simultaneous reconstruction of $\mu_{s}$ and $g$}

To date, the simultaneous reconstruction of $\mu_{s}$ and $g$-factor has not been considered in the literature for the optical imaging. In order to study and mime the crosstalk between the scattering parameters, two different test 
cases are considered. In this case, all the boundaries nodes are taken as detection locations for the inversion. For the first test, the inclusion varies only in anisotropy factor $\left(g^{*}=0.85\right)$ while it represents a heterogeneity only in scattering coefficient $\left(\mu_{s}^{*}=4 \mathrm{~mm}^{-1}\right)$ for the second test. The reconstructed results are depicted in Fig. 5. As shown, the reconstructed profile was spatially well fitted with the original distribution for the first test. The maximum local value retrieved in the $g$ inclusion has reached 0.84 against 0.85 . For the second test, the algorithm has localized the $\mu_{s}$ inclusion in its exact position but the quantitative accuracy was very underestimated. Indeed, the maximum value has reached only $2.5 \mathrm{~mm}^{-1}$ against $4 \mathrm{~mm}^{-1}$. The superiority in the quality reconstruction of the $g$-factor can be explained by the high sensitivity of this factor on the boundary data. It can be seen that the crosstalk effect induced in each parameter has been insignificant.

(a)

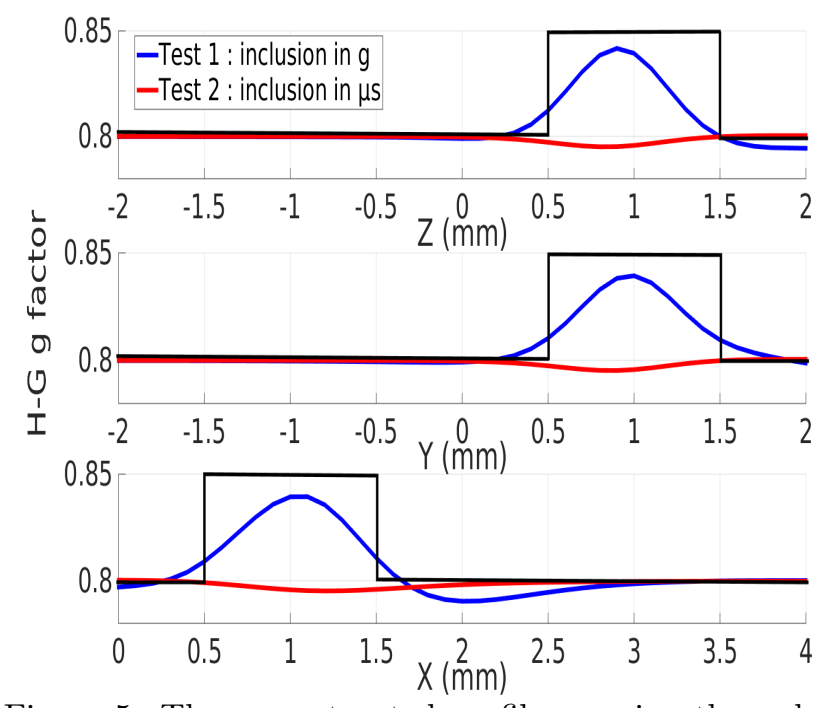

(b)

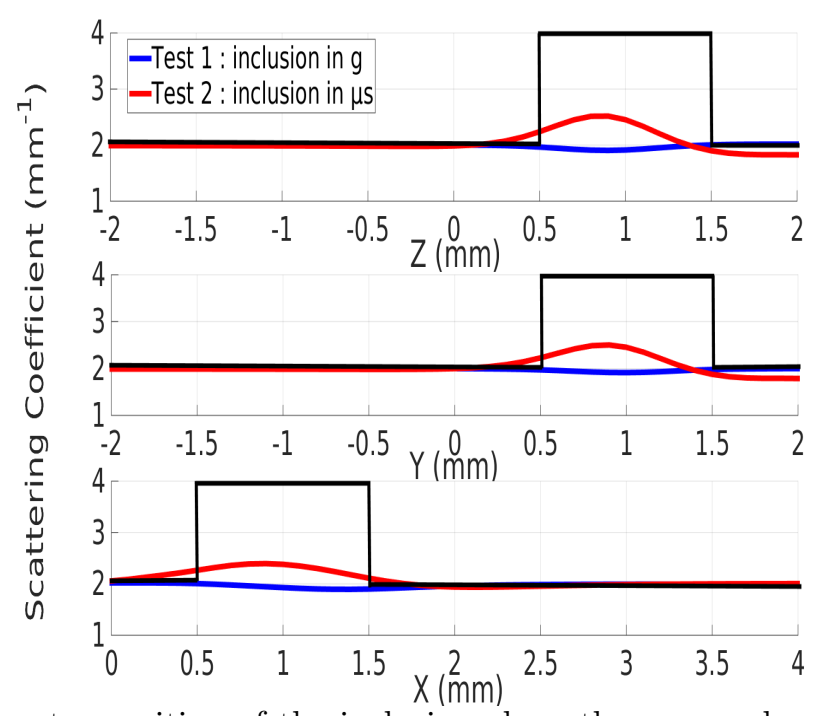

Figure 5: The reconstructed profiles passing through the center position of the inclusion along the $x, y$ and $z$ axis for the first test where the inclusion represents a heterogeneity only in $g$-factor and for the second one where the inclusion represents a heterogeneity only in $\mu_{s}$ coefficient : (a) anisotropy factor (b) scattering coefficient. The black solid profils represent the original position of the inclusion.

It should be noted that the reconstruction of $g$ would not have been possible with the Diffusion Equation since the anisotropy information is lost by considering the reduced scattering coefficient $\mu_{s}^{\prime}=\mu_{s}(1-g)^{11}$ where the $g$ value is given. Despite studies of 3D optical imaging were conducted with the RTE, none of these studies have presented the reconstruction of the $g$-factor, for the authors best knowledge. Although the example was carried out with synthetic data, it nevertheless constitutes an important first step (numerical validation) in the imaging of $g$-factor for optical imaging. This factor can be considered as a potential endogenous contrast which provides additional intrinsic information beside $\mu_{a}$ and $\mu_{s}$ for tumor diagnosis.

\section{CONCLUSION}

A 3D optical reconstruction algorithm based on the RTE-based forward model was presented to detect tumor-like inclusions. The main novelty of this work is the reconstruction of the spatial distribution of the anisotropy factor of the Henyey-Greenstein phase function. This was made possible thanks to the combination of the RTE-based forward model, the adjoint model, and the parallel computing. The algorithm is robust to recover and localize a heterogeneity in $g$-factor presenting low or high contrast level in the medium and with different initial guesses. The simultaneous reconstruction of $\mu_{s}$ and $g$ was reported for the time in DOT and was achieved with a reasonable quality. The crosstalk between the two parameters was weak. The parallel computing has led to considerably reduce the reconstruction times with a reasonable-order. This work was a necessary preliminary study before using the present algorithm with experimental boundary data and perform real small-tissues imaging. The simultaneous reconstruction of the three optical properties $\mu_{a}, \mu_{s}$ and $g$-factor is currently under investigation. 
That can be an important issue for optical tomography which it is not easy due to the ill-posed inverse problem and to the difference in nature, units, and sensitivities of the parameters on the boundary data.

\section{REFERENCES}

[1] Hillegersberg, R. V., Pickering, J. W., Maurice, A., and F., B. J., "Optical properties of rat liver and tumor at $633 \mathrm{~nm}$ and $1064 \mathrm{~nm}$ : Photofrin enhances scattering," Lasers in Surgery and Medicine 13(1), 31-39 (1993).

[2] Germer, C., Andr, R., Ritz, J. P., Christoph, I., Dirk, A., Gerhard, M., and J., B. H., "Optical properties of native and coagulated human liver tissue and liver metastases in the near infrared range," Lasers in Surgery and Medicine 23(4), 194-203 (1998).

[3] Yaroslavsky, A. N., Schulze, P. C., Yaroslavsky, I. V., Schober, R., Ulrich, F., and Schwarzmaier, H.-J., "Optical properties of selected native and coagulated human brain tissues in vitro in the visible and near infrared spectral range," Physics in Medicine \&3 Biology 47(12), 2059 (2002).

[4] Kim, H. K. and Charette, A., "A sensitivity function-based conjugate gradient method for optical tomography with the frequency-domain equation of radiative transfer," Journal of Quantitative Spectroscopy and Radiative Transfer 104(1), 24 - 39 (2007).

[5] Qiao, Y., Qi, H., Chen, Q., Ruan, L., and Tan, H., "An efficient and robust reconstruction method for optical tomography with the time-domain radiative transfer equation," Optics and Lasers in Engineering 78, 155 -164 (2016).

[6] Heino, J., Arridge, S., Sikora, J., and Somersalo, E., "Anisotropic effects in highly scattering media," Phys. Rev. E 68, 031908 (Sep 2003).

[7] Asllanaj, F., Contassot-Vivier, S., Liemert, A., and Kienle, A., "Radiative transfer equation for predicting light propagation in biological media: comparison of a modified finite volume method, the monte carlo technique, and an exact analytical solution," Journal of Biomedical Optics 19, 19 - 19 - 11 (2014).

[8] Addoum, A., Farges, O., and Asllanaj, F., "Optical properties reconstruction using the adjoint method based on the radiative transfer equation," Journal of Quantitative Spectroscopy and Radiative Transfer 204, 179 - 189 (2018).

[9] Kern, M., "Problèmes inverses : aspects numériques," Lecture (Sept. 2002).

[10] Elaloufi, R., Carminati, R., and Greffet, J.-J., "Diffusive-to-ballistic transition in dynamic light transmission through thin scattering slabs: a radiative transfer approach," J. Opt. Soc. Am. A 21, 1430-1437 (Aug 2004).

[11] Chu, M. and Dehghani, H., "Image reconstruction in diffuse optical tomography based on simplified spherical harmonics approximation," Opt. Express 17, 24208-24223 (Dec 2009). 